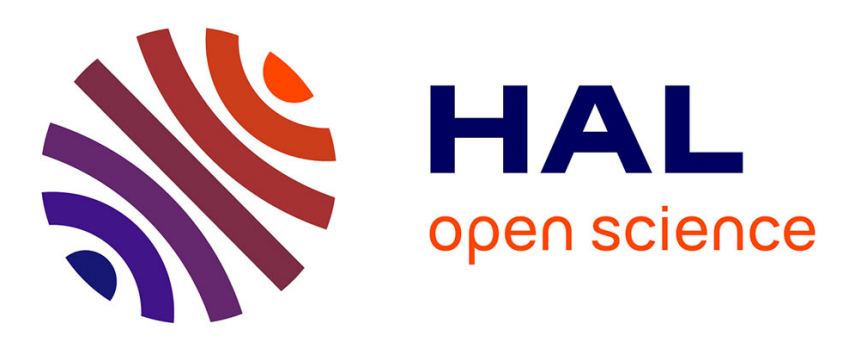

\title{
Sources de photons obtenus par annihilation en vol de positons
}

\author{
G. Audit, N. de Botton, G. Le Poitevin, C. Schuhl, G. Tamas
}

\section{To cite this version:}

G. Audit, N. de Botton, G. Le Poitevin, C. Schuhl, G. Tamas. Sources de photons obtenus par annihilation en vol de positons. Revue de Physique Appliquée, 1969, 4 (2), pp.293-294. 10.1051/rphysap:0196900402029300 . jpa-00243267

\section{HAL Id: jpa-00243267 https://hal.science/jpa-00243267}

Submitted on 1 Jan 1969

HAL is a multi-disciplinary open access archive for the deposit and dissemination of scientific research documents, whether they are published or not. The documents may come from teaching and research institutions in France or abroad, or from public or private research centers.
L'archive ouverte pluridisciplinaire HAL, est destinée au dépôt et à la diffusion de documents scientifiques de niveau recherche, publiés ou non, émanant des établissements d'enseignement et de recherche français ou étrangers, des laboratoires publics ou privés. 


\title{
SOURGES DE PHOTONS OBTENUS PAR ANNIHILATION EN VOL DE POSITONS
}

\author{
G. AUdit, N. De BOtTON, G. LE POITTEVIN, G. SCHUHL et G. TAMAS, \\ Service de Physique Nucléaire à Haute Énergie, G.E.N., Saclay.
}

\begin{abstract}
Résumé. - Nous présentons un calcul du spectre des photons obtenus par annihilation en vol de positons, tenant compte de la diffusion multiple et de la perte d'énergie des positons dans la cible d'annihilation. Nous donnons des résultats entre 150 et $450 \mathrm{MeV}$ (possibilités de l'Accélérateur Linéaire de Saclay) et les confrontons à des mesures à basse énergie.
\end{abstract}

Abstract. - We present a calculation of the spectrum of photons obtained from positron annihilation in flight; it takes account of multiple scattering and energy loss of positrons in the annihilation target. Results are presented in the range $150-450 \mathrm{MeV}$ (Saclay Linac) and compared with measurements at low energy.

I. Principe du calcul. - Un positon d'énergie initiale $\gamma_{0}=E_{+} / m_{0} c^{2} \quad\left(m_{0} c^{2}\right.$ : énergie au repos de l'électron) s'annihile après avoir traversé $m\left(\mathrm{~g} / \mathrm{cm}^{2}\right)$ de la cible $(Z, A)$ de masse totale $M$. Au moment de l'annihilation, il a perdu une partie de son énergie par collision; si $\varepsilon_{\text {coll. }}$ est le pouvoir de ralentissement du milieu, l'énergie du positon est alors en moyenne $\gamma=\gamma_{0}-\varepsilon_{\text {coll. }} m$. Par suite de la diffusion multiple, il a une probabilité $f_{\mathrm{D} \text { м. }}\left(\beta, \varphi_{\beta}\right)$ de se trouver à un angle $\left(\beta, \varphi_{\beta}\right)$ de sa direction initiale. Le photon d'annihilation émis vers l'avant dans le système du laboratoire a une direction $\left(\theta, \varphi_{\theta}\right)$ par rapport à la direction $\left(\beta, \varphi_{\beta}\right)$ du positon après diffusion multiple et une direction $\left(\alpha, \varphi_{\alpha}\right)$ par rapport à la direction initiale du positon. L'énergie du photon est :

$$
k=\frac{E_{\gamma}}{m_{0} c^{2}}=\frac{1+\gamma}{1+\gamma-\sqrt{\gamma^{2}-1} \cos \theta}
$$

et la section efficace différentielle du processus est $\mathrm{d} \sigma_{a} / \mathrm{d} \Omega(\theta)$ par électron de la cible.

La probabilité pour qu'un positon s'annihile dans la tranche $\mathrm{d} m$ en $m$, après avoir été diffusé suivant $\left(\beta, \varphi_{\beta}\right)$ dans $\mathrm{d} \Omega_{\beta}$, pour donner un photon dans $\mathrm{d} \Omega_{\theta}$ suivant la direction $\left(\theta, \varphi_{\theta}\right)$, est :

$$
\frac{\mathrm{d}^{5} F}{\mathrm{~d} \Omega_{\beta} \mathrm{d} \Omega_{\theta} \mathrm{d} m}=f_{\text {D.M. }}(\beta) \frac{N Z}{A} \frac{\mathrm{d} \sigma_{a}}{\mathrm{~d} \Omega_{\theta}} .
$$

Nous avons utilisé la section efficace différentielle d'annihilation en deux photons au premier ordre et négligé les corrections radiatives à ce processus ainsi que l'annihilation en trois photons. De plus, nous avons fait l'approximation des petits angles $(\theta \ll 1)$. Nous avons assimilé la distribution de la diffusion multiple à une gaussienne d'angle caractéristique $\bar{\beta}$. Deux déterminations de $\bar{\beta}^{2}$ ont été employées' : i) la première correspond au traitement de Williams [1]; ii) dans un second calcul, nous avons utilisé pour $\bar{\beta}^{2}$ l'expression donnée par Scott [2] à partir de la formule de Molière et nous avons tenu compte de la diffusion inélastique sur les électrons par la correction de Fano. Cette manière plus rigoureuse de traiter la diffusion multiple n'a entraîné que des modifications de l'ordre du $\%$ tant sur le spectre que sur le nombre de photons d'annihilation. Après intégration sur $\varphi$ et $\alpha, \operatorname{si} P\left(\chi^{\prime 2} \mid \nu, \lambda\right)$ est la fonction de distribution du $\chi^{2}$ non central de paramètre de non-centralité $\lambda$ à $\nu$ degrés de liberté, le spectre des photons pour un positon incident d'énergie $\gamma_{0}$ sera donné par :

$\frac{\mathrm{d} F}{\mathrm{~d} k}=2 \pi \frac{N Z}{A} \int_{0}^{\mathrm{M}} \mathrm{d} m \frac{\mathrm{d} \sigma_{a}(k, m)}{\mathrm{d} k \mathrm{~d} \varphi} P\left[\frac{2 a^{2}}{\bar{\beta}^{2}(m)} \mid 2,2 \frac{\theta^{2}(k, m)}{\bar{\beta}^{2}(m)}\right]^{+}$.

En fait, les positons sont distribués en énergie suivant un spectre $g\left(\gamma_{0}\right)$ normalisé à l'unité. Le spectre des photons pour un positon incident est :

$$
\frac{\mathrm{d} N}{\mathrm{~d} k}=\int g\left(\gamma_{0}\right) \frac{\mathrm{d} F}{\mathrm{~d} k}\left(k, \gamma_{0}\right) \mathrm{d} \gamma_{0} .
$$

II. Résultats du calcul. - Lorsque le matériau de la cible est choisi, la raie de photons dépend encore de 5 paramètres : l'épaisseur de la cible, l'angle solide d'acceptance des photons, l'énergie, le spectre et l'émittance du faisceau de positons. L'utilisation de ce calcul doit permettre de déterminer, pour une expérience donnée, les valeurs optimales de ces différents paramètres. A titre d'exemple, nous avons représenté ( fig. 1) les spectres d'annihilation et de freinage, à $300 \mathrm{MeV}$, dans un angle $a=4 \times 10^{-3}$, sur une cible d'hydrogène liquide de $0,42 \mathrm{~g} / \mathrm{cm}^{2}$ comportant deux fenêtres 


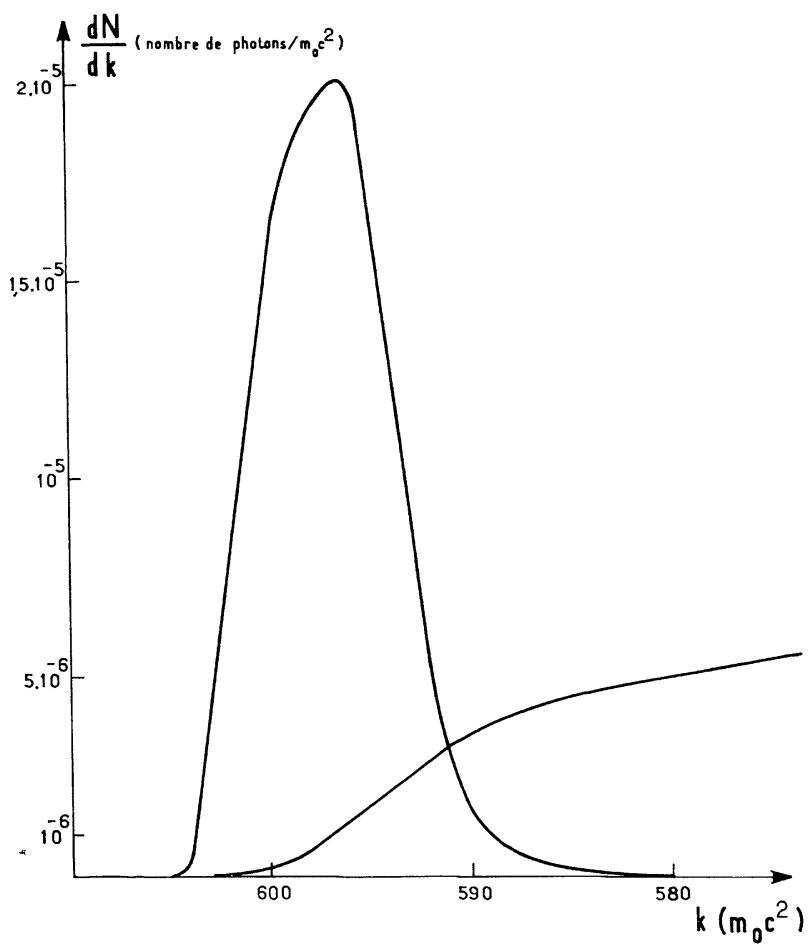

FIG. 1. - Spectres d'annihilation et de freinage calculés pour une cible d'hydrogène : $E_{+}=600 m_{0} c^{2} ; \Delta E_{+}$ $=8 m_{0} c^{2}$ rectangle ; $a=4 \times 10^{-3} ; \mathrm{H}_{2} 0,42 \mathrm{~g} / \mathrm{cm}^{2}$ +2 parois $5 / 100 \mathrm{~mm} \mathrm{Al} ; 1,68 \times 10^{-4}$ photon annihilation/positon incident.

d'aluminium de $0,05 \mathrm{~mm}$ chacune. Ces conditions expérimentales seront celles utilisées dans les premières expériences à l'Accélérateur Linéaire de Saclay.

III. Etude expérimentale de la source d'annihilation de l'accélérateur linéaire de $45 \mathrm{MeV}$ de Saclay.

A l'aide de cet appareil, nous avons étudié le nombre de photons et la forme de la raie d'annihilation. Nous avons obtenu le nombre de photons d'annihilation par positon incident en plaçant un cristal de INa directement dans le faisceau et en soustrayant les spectres obtenus en positons et en négatons et, ce, pour quatre énergies. L'accord expérience-calcul est excellent quant à l'allure de la variation, mais il y a un écart systématique, de l'ordre de $10 \%$, sur les valeurs absolues qui pourrait s'expliquer par l'incertitude sur le rendement calculé de la chambre d'ionisation à xénon mesurant le courant de positons.

Les points expérimentaux ont été obtenus en détectant, à l'aide de deux cristaux de $\mathrm{INa}$, les photons diffusés par une cible de carbone. En faisant varier l'énergie des positons, on déplace la raie de photons par rapport au niveau très étroit $(70 \mathrm{eV})$ de $15,11 \mathrm{MeV}$ de ${ }^{12} \mathrm{G}$. La série des dix mesures effectuées donne ainsi l'image du spectre des photons (isochromate). L'accord satisfaisant de la forme du spectre calculé avec celle du spectre expérimental (fig. 2) pourrait probablement

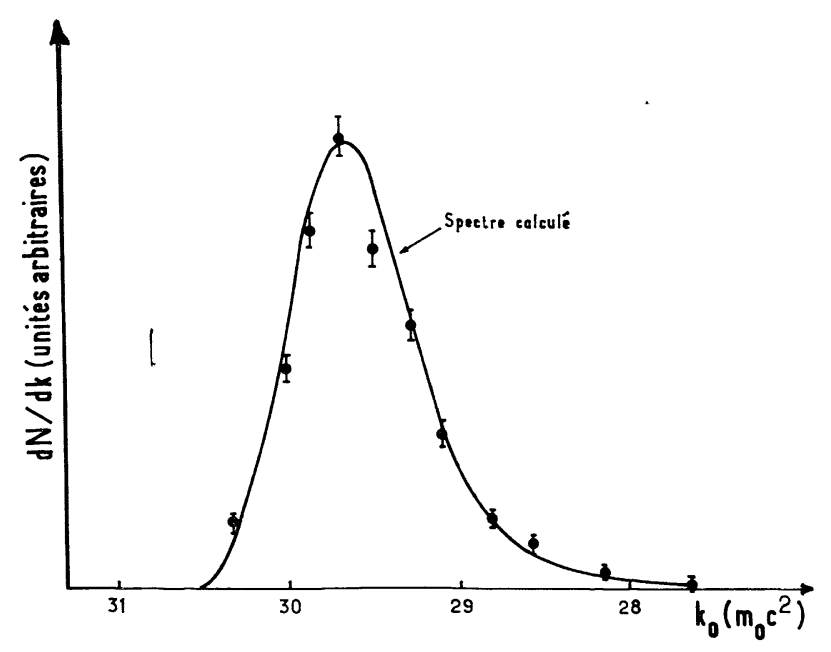

FIG. 2. - Isochromate de la raie d'annihilation obtenue par diffusion sur ${ }^{12} \mathrm{C}^{*}(11,5 \mathrm{MeV}): \quad \mathbf{I}=$ Points expérimentaux ; $E_{+}=29,57 m_{0} c^{2}$; Résolution triangulaire (2\% à mi-hauteur) ; $m=0,17 \mathrm{~g} / \mathrm{cm}^{2}$ de $\mathrm{Li} ; a=$ $0,0226 \mathrm{rad}$.

être amélioré en introduisant dans le calcul une forme de spectre énergétique des positons plus réaliste que la forme triangulaire déduite en première approximation de l'optique du système de transfert du faisceau.

\section{BIBLIOGRAPHIE}

[1] Wilimiams (E. J.), Proc. Roy. Soc. (London), 1939, A 169, 531.

[2] Scoti (W. T.), Rev. Mod. Phys., 1963, 35, 231. 